\title{
Biological Effects of Targeted Inactivation of Hepatocyte Growth Factor-like Protein in Mice
}

\author{
Jorge A. Bezerra, ${ }^{\star}$ Terri L. Carrick, ${ }^{\star}$ Jay L. Degen,, David Witte, ${ }^{\S}$ and Sandra J.F. Degen ${ }^{\ddagger}$ \\ $*$ Division of Gastroenterology and Nutrition, ${ }^{*}$ Division of Developmental Biology, and ${ }^{\S}$ Division of Pathology, Children’s Hospital \\ Research Foundation and Department of Pediatrics, University of Cincinnati, Cincinnati, Ohio 45229-3039
}

\begin{abstract}
Hepatocyte growth factor-like protein (HGFL) is a liverderived serum glycoprotein involved in cell proliferation and differentiation, and is proposed to have a fundamental role in embryogenesis, fertility, hematopoiesis, macrophage activation, and tissue repair. To assess the in vivo effects of total loss of HGFL, we generated mice with targeted disruption of the gene resulting in loss of the protein. Disruption of the HGFL gene allowed for normal embryogenesis, and followed a Mendelian pattern of genetic transmission. Mice homozygous for the targeted allele $\left(\mathrm{HGFL}^{-1-}\right.$ mice) are fertile, and grow to adulthood without obvious phenotypic abnormalities in unchallenged animals, except for development of lipid-containing cytoplasmic vacuoles in hepatocytes throughout the liver lobules. These histologic changes are not accompanied by discernible changes in synthetic or excretory hepatic functions. Hematopoiesis appears unaltered, and although macrophage activation is delayed in the absence of HGFL, migration to the peritoneal cavity upon challenge with thioglycollate was similar in $\mathrm{HGFL}^{-/-}$and wild-type mice. Challenged with incision to skin, $\mathrm{HGFL}^{-1-}$ mice display normal wound healing. These data demonstrate that HGFL is not essential for embryogenesis, fertility, or wound healing. HGFL-deficient mice will provide a valuable means to assess the role of HGFL in hepatic and systemic responses to inflammatory and infectious stimuli in vivo. (J. Clin. Invest. 1998. 101:1175-1183.) Key words: HGFL • MSP • HGF • growth factor • macrophages
\end{abstract}

\section{Introduction}

Consistent with their fundamental role in directing cell proliferation and differentiation, polypeptide growth factors, cytokines, and hormones have frequently been shown to be critical in embryogenesis, growth to adulthood, and disease pathogenesis. One unusual family of growth factors whose biological properties and physiological significance remain to be fully defined is the hepatocyte growth factor $(\mathrm{HGF})^{1} /$ scatter factor and $^{2}$ hepatocyte growth factor-like protein (HGFL) family. These growth factors/cytokines are distinguished by the presence of

Address correspondence to Jorge A. Bezerra, Division of Gastroenterology and Nutrition, Children's Hospital Medical Center, 3333 Burnet Avenue, Cincinnati OH 45229. Phone: 513-636-4415; FAX: 513-636-7805; E-mail: jorge.bezerra@chmcc.org

Received for publication 18 September 1997 and accepted in revised form 29 December 1997.

J. Clin. Invest.

(C) The American Society for Clinical Investigation, Inc. 0021-9738/98/03/1175/09 \$2.00

Volume 101, Number 5, March 1998, 1175-1183

http://www.jci.org both kringle domains and a proteolytically nonfunctional serine protease-like domain, structures similar to domains frequently found in coagulation (e.g., prothrombin and factor XII) and fibrinolytic (e.g. plasminogen and plasminogen activator) factors (1-4). Although HGF has been shown to be essential for embryogenesis and to have mitogenic, morphogenic, and migratory properties on a tissue-specific basis, the biological role for HGFL remains largely unknown (5-9).

HGFL is synthesized in the liver and secreted into the plasma as an $80-95 \mathrm{kD}$ single-chain protein $(4,10-12)$. It undergoes activation by serum proteases of the intrinsic coagulation cascade (including kallikrein, factor XIa, and factor XIIa), nerve growth factor- $\gamma$, epidermal growth factor-binding protein, and membrane-bound proteases to form a heterodimeric, mature protein containing a heavy chain of $65 \mathrm{kD}$ linked to a light chain of $30 \mathrm{kD}$ by a disulfide bond (13-15). Analysis of the translated amino acid sequence and use of recombinant protein have shown that HGFL is identical to macrophagestimulating protein $(3,4,10,12)$. HGFL is an inflammatory cytokine able to activate macrophages and to interact with other inflammatory cytokines $(3,16,17)$. Recent studies have broadened the functional spectrum of HGFL to include the ability to stimulate proliferation of mammary duct epithelial cells and keratinocytes, maturation of megakaryocytes, motility of keratinocytes, and bone resorption and contraction of osteoclasts (18-21). HGFL also acts as an inducer of adhesion and apoptosis of pulmonary neuroendocrine cells, suggesting that the protein might regulate cell survival during preneoplastic lung injury (22). Based on these in vitro studies, HGFL has been proposed to participate in a wide spectrum of biological processes such as inflammation, tissue remodeling/wound healing, hematopoiesis, and bone formation.

The receptor for HGFL has been identified as the product of the human protooncogene ron and of its murine counterpart stk, members of a tyrosine kinase family of receptors that includes the product of the protooncogene met, the receptor for HGF $(18,23-25)$. The product of ron/stk is a heterodimeric membrane-anchored protein with a ligand-binding extracellular domain and an intracellular motif with tyrosine kinase activity that initiates intracellular signal transduction channeled through a multifunctional binding site $(18,26)$. HGFL has been shown to bind to $\mathrm{ron} / \mathrm{st}$, inducing proliferation, migration, invasion, and polarization in both mesenchymal and epithelial cells $(18,24,27)$. Although ron/stk is not active without the ligand, cellular overexpression of the receptor and a splicing variant present in gastric carcinoma cells allow constitutive tyrosine kinase activity and an invasive phenotype, properties

1. Abbreviations used in this paper: ES, embryonic stem; HGF, hepatocyte growth factor; HGFL, hepatocyte growth factor-like protein; hprt, hypoxanthine phosphoribosyl transferase; HSV-tk, herpes simplex virus thymidine kinase; pgk, phosphoglycerate kinase. 
that imply that HGFL may also play an important role in carcinogenesis $(18,28)$.

The shared structural identity with $\mathrm{HGF}$ and the mRNA expression for both HGFL and stk point toward a role of HGFL in embryogenesis as demonstrated for its homolog $\operatorname{HGF}(5,6,29)$. To assess the role of HGFL in embryogenesis and the wide spectrum of biological properties attributed to the protein, we used gene targeting to assess the function of HGFL in vivo. We report that HGFL-deficient mice develop to term, grow to adulthood without obvious phenotypic abnormality despite development of cytoplasmic vacuolization of hepatocytes, display efficient wound repair, and produce and sustain offspring.

\section{Methods}

Reagents and instruments. The pBluescript $\mathrm{SK}^{\mathrm{TM}}(+/-)$ plasmid was obtained from Stratagene (La Jolla, CA) while RPMI 1640 and Hanks' buffered salt solution were supplied by GIBCO BRL (Grand Island, NY). Electroporation was performed with the GeneZapper (IBI, Madison, WI). Thioglycollate medium (4\%) was from Becton Dickinson Microbiology Systems (Cockeysville, MD), 10\% formalin fixative was from Accra Laboratory (Swedesboro, NJ), dextran sulfate sodium powder of 40,000-50,000 $\mathrm{kD}$ was from Amersham Corp. (Arlington Heights, IL), and Hemoccult ${ }^{\mathrm{TM}}$ cards were from Smithkline Diagnostics Inc. (San Jose, CA). The sedatives ketamine and xylazine were obtained from Phoenix Pharmaceutical Inc. (St. Joseph, MO), acepromazine maleate was from Fort Dodge Laboratories, Inc. (Fort Dodge, IA), and isofluorane was from Mallinckrodt Veterinary Inc. (Mundelein, IL). Peritoneal cells were counted using a Counter ZM (Coulter Electronics Limited, England), while blood cell counts and hematocrit were established using a Cell-Dyn 3500 blood cell analyzer (Abbot Laboratories Diagnostic Division, Chicago, IL), and biochemical markers of liver function were determined by an automated enzymatic assay using the Vistros Chemistry Systems 950 (Johnson \& Johnson, Rochester, NY). Signal detection in Western analysis was performed with the antirabbit Vistra enhanced ECF Western blot reagent pack obtained from Amersham Corp. Radioisotope- and chemifluorescent-based signals were detected using phosphor screens and an ImageQuant phosphorimager (Molecular Dynamics, Inc., Sunnyvalle, CA). Values are shown as mean \pm SD, and statistical significance was assessed by unpaired $t$ test, with a significance level at $P<0.05$.

Construction of the targeting vector. Two fragments isolated from the mouse gene coding for HGFL (the gene was isolated from a 129 SvJ genomic DNA library) were cloned into the same pBluescript $\mathrm{SK}^{\mathrm{TM}}(+/-)$ plasmid. These fragments included a $1.2-\mathrm{kb}$ Xho I fragment coding for exons 1 through part of exon 3, and a 3.4-kb BamH I-Sst I fragment coding for part of exon 8 through exon 18 plus the $3^{\prime}$ flanking region. A $3.2-\mathrm{kb}$ Sal I fragment coding for the mouse hypoxanthine phosphoribosyl transferase (hprt)-selectable marker expressed under the control of the phosphoglycerate kinase ( $p g k)$ promoter (30) was cloned between the two HGFL gene fragments at the Sal I site of pBluescript $\mathrm{SK}^{\oplus}(+/-)$. To provide a means of selection against cells that randomly insert the targeting vector into the genome (31), a 6.0-kb Kpn I fragment of this initial construct (encompassing both gene fragments separated by the hprt minigene) was subcloned into another pBluescript SK ${ }^{\mathrm{TM}}(+/-)$ containing a 2-kb Cla I cassette encoding the herpes simplex virus thymidine kinase (HSV-tk) gene. Because this subcloning step shortened the length of the DNA fragment coding for the $3^{\prime}$ end of the gene, the targeting vector was further modified by adding a 1.2-kb DNA fragment from the $3^{\prime}$ end of the HGFL gene, which reconstituted part of exon 8 through exon 18 plus $260 \mathrm{bp}$ of the $3^{\prime}$ flanking region. A $8.8-\mathrm{kb}$ Not I fragment was used to electroporate embryonic stem (ES) cells (see Fig. 1).

Generation of targeted mice. The targeting vector was introduced into the hprt-deficient ES cell line E14TG2a (32) by electroporation $(800 \mathrm{~V} / \mathrm{cm}$ and $200 \mu \mathrm{F})$ of $10^{8}$ cells resuspended in $1 \mathrm{ml}$ of Hanks' buffered salt solution, containing $50 \mu \mathrm{g}$ of the vector in a $0.4-\mathrm{cm}$ cuvette, and stable transfectants were selected as described previously (33). Screening for targeted ES cell clones was initially done by PCR analysis of genomic DNA using diagnostic primers A and C (see Fig. 1) that amplify a predicted 1,250-bp product only in the presence of the targeted allele as a template. Primer A (5'-GGGACACCTAACGTTTCCACA-3', corresponding to nucleotides -171 to -151 in the mouse HGFL gene sequence; 2) is complementary to a sequence upstream of the gene that was not included in the targeting vector, and primer C (5'-GGAAAAGCGCCTCCCCTACCCGG-3'; 34 ) is complementary to a sequence in the hprt minigene cassette. Incorporation of the targeting vector sequences by homologous recombination was further demonstrated in selected clones by Southern analysis (35) using Bgl II as a diagnostic restriction enzyme and hybridization with a 250-bp Kpn I-Xho I fragment of the HGFL gene (see Fig. 1). ES cells found to have one targeted HGFL allele were injected into the blastocoel cavity of $\mathrm{C} 57 \mathrm{Bl} / 6$-derived blastocysts and implanted into pseudopregnant female mice at the ES Cell Altered Mouse Service Core (University of Cincinnati; 35). Chimeric offspring were bred to National Institutes of Health Swiss Black mice to generate hemizygous offspring, which were then interbred to homozygozity for the targeted allele. All mice were maintained in a specific pathogen-free facility and routinely monitored for the absence of common mouse viruses by serology. Animal protocols were approved by the Institutional Animal Care and Use Committee of the Children's Hospital Research Foundation (Cincinnati, OH).

Genotype analysis. Genotypes of mice were determined using tail biopsy DNA (35) by Southern analysis and PCR. Southern analysis of Bgl II digests of tail DNA was performed as described above. In PCR assays, mice carrying one or two targeted alleles were identified using a multiplex reaction mixture containing primers $\mathrm{A}$ and $\mathrm{C}$ as above, and primer B (5'-CGCCCTGACTCTGTAACGTC-3', corresponding to nucleotides +1658 to +1677 of the mouse $H G F L$ gene; 2) which is complementary to a sequence in exon 6 of the native allele, and together with primer A amplifies a product of $1841 \mathrm{bp}$.

Northern and Western analysis. Total RNA from the liver was isolated, size-fractionated, transferred to a nylon membrane, and hybridized with a ${ }^{32} \mathrm{P}$-labeled 1.4-kb Eco RI mouse $H G F L$ cDNA fragment as previously described (11). Western analysis was performed with $0.5-\mu l$ aliquots of mouse sera incubated with a previously characterized rabbit polyclonal antibody that recognizes the single chain form of HGFL (10), and specific signal was detected by chemifluorescence.

Histological survey and liver function tests. Tissues collected for histological analyses were fixed overnight in formalin, embedded in paraffin, sectioned, and stained with hematoxylin/eosin. Staining with periodic acid Schiff (for carbohydrate) and oil red-o and Sudan black (for lipid) were performed on frozen liver sections according to standard methods. Transmission electron microscopy studies were performed in liver samples harvested into cold 3\% glutaraldehyde in $0.175 \mathrm{M}$ sodium cacodylate solution, $\mathrm{pH} 7.4$, postfixed and stained by routine techniques, and examined on a Ziess 100 transmission electron microscope. For hematological analyses, bone marrow cells were obtained from the femur of 1-mo-old mice after complete removal of the femur, a small incision in the patellar condyle, brief centrifugation into an Eppendorf tube, and fixation of the bone marrow effluent cells in $4 \%$ paraformaldehyde at $4^{\circ} \mathrm{C}$ overnight. Cells were then cytospun onto a glass slide, stained with hematoxylin/eosin, and examined under light microscopy. Circulating blood cells were analyzed in blood samples collected from the inferior vena cava of mice anesthetized with $0.1 \mathrm{ml} / 30 \mathrm{~g}$ body wt of ketamine/xylazine/acepromazine (4:1:1), and blood cell counts and hematocrit were established using the automated Cell-Dyn 3500 blood cell analyzer (Abbot Laboratories Diagnostic Division). Determination of serum albumin, aminotransferases, bilirubin, gamma-glutamyltranspeptidase, and alkaline phosphatase was performed by an automated enzymatic assay with the Vistros Chemistry Systems 950 (Johnson \& Johnson). 
Activation of peritoneal macrophages. Assessment of in vitro activation of peritoneal macrophages was performed as previously described $(3,4,12,16)$. In brief, macrophages were obtained by peritoneal lavage with 7-8 $\mathrm{ml}$ of RPMI 1640 medium, centrifuged at $4^{\circ} \mathrm{C}$ for 3 min at $50 \mathrm{~g}$, resuspended in RPMI 1640, and plated in 24-well dishes at a density of $5 \times 10^{5}$ cells/well. After a 30 -min incubation at $37^{\circ} \mathrm{C}$ in humidified air with 5\% $\mathrm{CO}_{2}$, the wells were washed with RPMI 1640 and incubated with RPMI 1640 with or without $10 \%$ serum from wild-type or homozygous null mice. At different time points, the number of activated macrophages were counted per high power field $(200 \times)$ using a reverse-phase microscope. Activation was defined by formation of cytoplasmic elongations and condensed nuclei in adherent macrophages $(16,12)$. For isolation of in vivo-stimulated peritoneal macrophages, 4-6-wk-old mice were injected intraperitoneally with $1 \mathrm{ml}$ of $4 \%$ thioglycollate, and macrophages were obtained from peritoneal lavage after $3 \mathrm{~d}$. The total number of peritoneal exudate cells was counted using the Counter ZM (Coulter Electronics Limited). Cells were washed and plated in RPMI 1640 medium. At 30 min of incubation, the nonadherent cells were removed with gentle washing with RPMI 1640, and the number of activated macrophages was counted immediately and expressed as a percentage of the total number of adhered macrophages for each mouse.

Wound healing and induction of acute colitis. For wound healing experiments, full-thickness skin incisions were performed with a surgical scalpel in the interscapular region of 6-wk-old mice anesthetized with $2 \%$ isoflurane. Incisional wounds were $15 \mathrm{~mm}$ long with a depth to expose the underlying fascia $(\sim 2 \mathrm{~mm})$, and they were neither dressed nor sutured (36). The mice were caged individually, and the incisions were examined visually on a daily basis after surgery. For studies of chemically induced colitis, $5 \%$ dextran sulfate sodium in tap water ( wt/vol) was administered orally for $7 \mathrm{~d}$ as the source of drinking water $(37,38)$. Evaluation of clinical colitis was determined by daily weights, examination of stool consistency, and the presence of visible or microscopic blood using Hemoccult ${ }^{\mathrm{TM}}$ cards. Mice were killed at day 7, and the small intestine and colon were removed and cleaned briefly with $137 \mathrm{mmol} /$ liter $\mathrm{NaCl}, 2.7 \mathrm{mmol} /$ liter $\mathrm{KCl}, 4.3$ $\mathrm{mmol} /$ liter $\mathrm{Na}_{2} \mathrm{HPO}_{4} .7 \mathrm{H}_{2} 0$, and $1.4 \mathrm{mmol} /$ liter $\mathrm{KH}_{2} \mathrm{PO}_{4}(\mathrm{pH} 7.4 ; \mathrm{PBS})$. $1-\mathrm{cm}$ sections were paraffin-embedded for histological examination as above.

\section{Results}

Generation of HGFL-deficient mice. To generate mice lacking the HGFL gene product, the targeting vector shown in Fig. 1 was introduced into hprt-deficient ES cells by electroporation. Southern analysis and PCR of genomic DNA extracts identified one targeted clone (no. 224) out of 306 stable transfectants isolated for analysis that had incorporated the targeting vector by homologous recombination, containing one copy of the endogenous allele and one copy of the targeted allele (Fig. 2, $A$ and $B$ ). Targeted ES cells were used to generate 17 chimeric founder mice, three of which were found to have transmitted the mutant $H G F L$ allele to their offspring when crossed with National Institutes of Health Swiss Black mice.

Viability of $\mathrm{HGFL}^{-/-}$mice. Hemizygotes $\left(\mathrm{HGFL}^{+/-}\right)$were backcrossed once to National Institutes of Health Swiss Black mice, and their hemizygous offspring were intercrossed to generate mice homozygous $\left(\mathrm{HGFL}^{-/-}\right)$for the targeted allele as demonstrated by PCR and Southern analysis (Fig. 2, $C$ and $D$ ). Genomic analysis of 377 offspring showed that the loss of HGFL is compatible with development to term and survival beyond the neonatal period. Furthermore, transmission of the mutant HGFL allele followed a Mendelian pattern of inheritance,

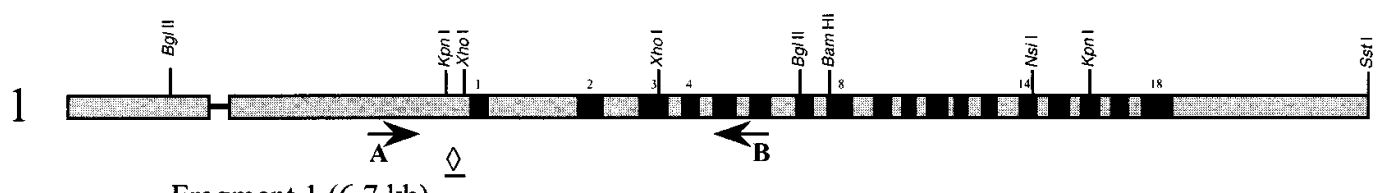

Fragment $1(6.7 \mathrm{~kb})$

2

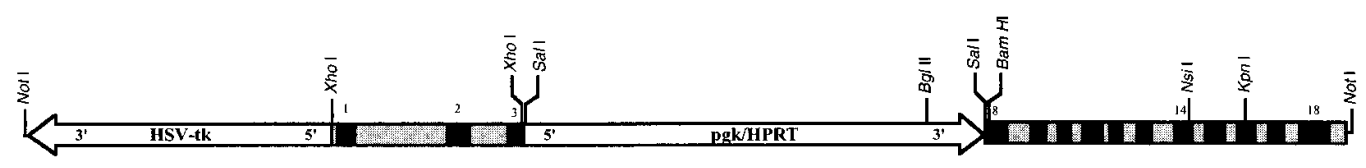

3

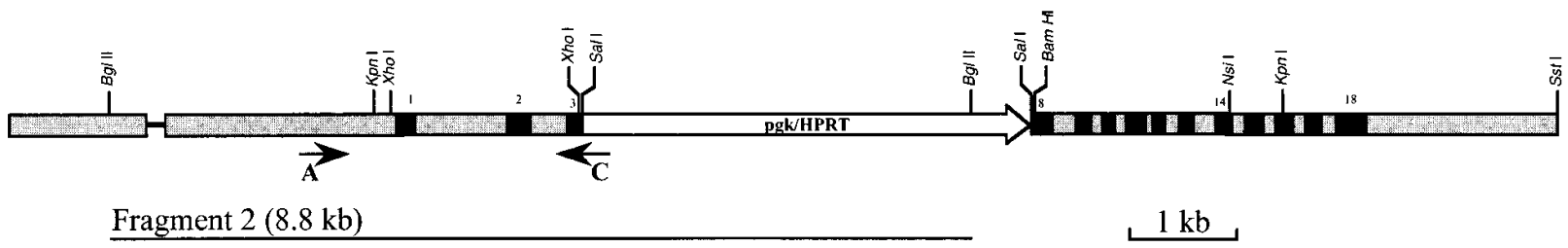

Figure 1. Strategy to target the mouse HGFL gene by homologous recombination. Structural organization of the (1) normal mouse HGFL gene, (2) targeting vector used to electroporate ES cells, and (3) the targeted HGFL gene. Solid bars indicate the positions of exons, and are numbered 1-18. The 1023-bp Xho I-Bam HI fragment encompassing part of exon 3 through the beginning of exon 8 was replaced by a 3.2-kb Sal I fragment coding for the mouse $\mathrm{pgk} / \mathrm{hprt}$ selectable marker (open bar), which was cloned in the same orientation as the HGFL gene relative to the direction of transcription. To select against random insertion of the targeting vector into the genome, a 2-kb Cla I cassette encoding the HSV-tk gene was cloned at position -82 in the gene sequence (2). The PCR oligonucleotide primers and the Southern blot hybridization probe used to screen the ES cell clones are shown below 1 and 3. Primers A (nucleotides -171 to -151 of the mouse HGFL gene; 2) and B (nucleotides +1658 to +1677 of the mouse $H G F L$ gene; 2 ) amplify a 1841-bp PCR product using the endogenous allele as a template, whereas primers A and C (unique to the $\mathrm{pgk} / \mathrm{hprt}$ gene; 33) amplify a 1250-bp product in the presence of the targeted allele. A 250-bp Kpn I-Xho I DNA fragment ( $\diamond)$ present only in the endogenous allele hybridizes to a $6.7-\mathrm{kb}$ Bgl II fragment in the endogenous allele and a 8.8-kb Bgl II fragment in the targeted allele as determined by Southern analysis. 
A


Figure 2. Identification of the targeted HGFL gene. (A) Targeting of the $H G F L$ gene by homologous recombination is demonstrated by Southern analysis of Bgl II digest of DNA from ES cell clones using a 250-bp Kpn I-Xho I DNA fragment specific to the endogenous gene (see Fig. 1). The fragment hybridizes to the 6.7-kb endogenous allele in two control clones (no. 17 and 222) and to the $6.7-\mathrm{kb}$ endogenous allele in addition to the $8.8-\mathrm{kb}$ targeted allele in clone no. 224. (B) Successful targeting of clone no. 224 is also shown by $3^{\prime}$ PCR amplification of the 1841-bp product from the endogenous allele in addition to the 1,250-bp product from the targeted allele (only the endogenous allele is seen in clone no. 222). Representative PCR $(C)$ and Southern analysis $(D)$ are shown identifying mice carrying the targeted disruption of the $H G F L$ gene using tail biopsy DNA prepared from wildtype $(+/+)$, hemizygous HGFL $(+/-)$, and homozygous HGFL $(-/-)$ mice generated from ES cell clone no. 224. with 91 (24\%) $\mathrm{HGFL}^{+/+}, 177$ (47\%) $\mathrm{HGFL}^{+/-}$, and $109(29 \%)$ $H G F L^{-/-}$mice identified. Based on the known liver-specific expression of HGFL mRNA (11), we performed Northern analysis using total RNA isolated from the liver of wild-type, hemizygous, and null mice. No detectable HGFL mRNA was found in $\mathrm{HGFL}^{-1-}$ mouse liver, while the level of HGFL mRNA in the liver of $H G F L^{+/-}$littermates was approximately half of that found in wild-type mice (Fig. $3 A$ ). Since HGFL is constitutively expressed and present in the serum, we assessed the systemic loss of HGFL by analyzing sera of targeted mice by Western analysis. Consistent with the absence of HGFL mRNA, no HGFL can be detected in the serum of $H G F L^{-/-}$ mice (Fig. 3 B). Thus, based on analyses of HGFL expression at both the mRNA and protein levels, the strategy used for disrupting the HGFL gene in mice appears to result in an $H G F L$ null phenotype. Nevertheless, no obvious phenotypic abnormalities were observed in unchallenged $H G F L^{-1-}$ mice.

Microscopic analyses of $\mathrm{HGFL}^{-1-}$ mice. To examine in more detail the impact of the loss of HGFL in vivo, we performed histological analyses on tissues collected from $\mathrm{HGFL}^{-/-}$mice at 1-6 mo of age. All organs appeared normal except for the liver, which showed varying degrees of cytoplasmic vacuolization of hepatocytes without inflammation or changes in the bile duct or other nonparenchymal cells (Fig. 4). Cytoplasmic vacuolizations were present in $79 \%$ of $H G F L^{-/-}$mice, and were distributed uniformly throughout the liver lobule. These vacuolizations were present at 1 mo of age and did not appear to progress up to 6 mo of age, although in some livers the lesion was severe. Staining with periodic acid Schiff (for carbohydrates) was negative, but oil red-o and Sudan black demonstrated that the vacuoles contained lipid (Fig. $4 E$ ). Although transmission electron miscroscopy showed mostly intact organelles, there was some membrane debris and clear droplets in the cytoplasm not surrounded by membranes (Fig. $4 F$ ). Macroscopically, livers appeared normal, and serum biochemical indicators of hepatic synthetic and excretory functions (serum concentration of albumin, bilirubins, aminotransferases, gamma-glutamyltranspeptidase, and alkaline phosphatase) were normal in $\mathrm{HGFL}^{-/-}$mice, indicating that these vacuoles did not appear to compromise liver function. Thus, despite the previously proposed role for HGFL in embryogenesis, the only
$\mathbf{A}$

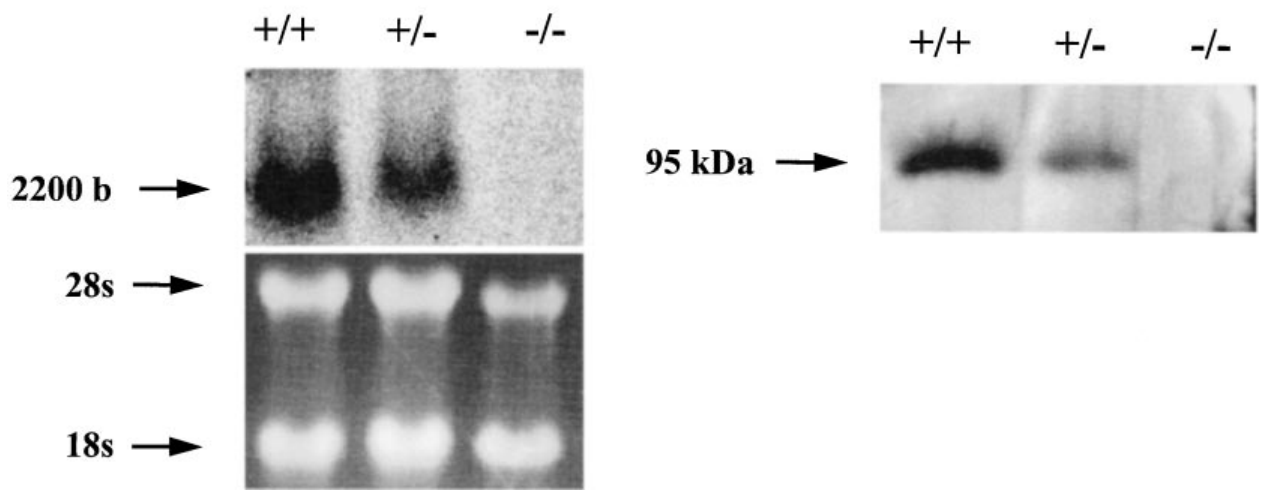

Figure 3. Identification of mice carrying the targeted loss of $H G F L$.

Representative Northern and Western analyses of offspring from mating hemizygous mice demonstrating the targeted loss of HGFL. (A) Total RNA from the livers of wild-type $(+/+)$, hemizygous $H G F L(+/-)$, and homozygous $H G F L(-/-)$ mice hybridized with a 1,400-bp Eco RI $H G F L$ cDNA fragment shows that specific expression is reduced by $\sim 50 \%$ in the $H G F L^{+/-}$liver when compared with the specific signal in the $H G F L^{+/+}$liver, and is absent in the $H G F L^{-1-}$ liver. Ethidium bromide staining of $28 \mathrm{~s}$ and $18 \mathrm{~s}$ ribosomal RNA is included to show

loading of total RNA in each lane. $(B)$ Sera $(0.5 \mu l)$ from the same mice as in $A$ analyzed by Western analysis under reducing conditions using a polyclonal antibody that recognizes the single-chain and alpha (heavy)-chain forms of HGFL show complete loss of the protein in $H G F L^{-/-}$serum, while approximately half of the specific signal in the $H G F L^{+/+}$serum is seen in the lane containing $H G F L^{+/-}$serum. 

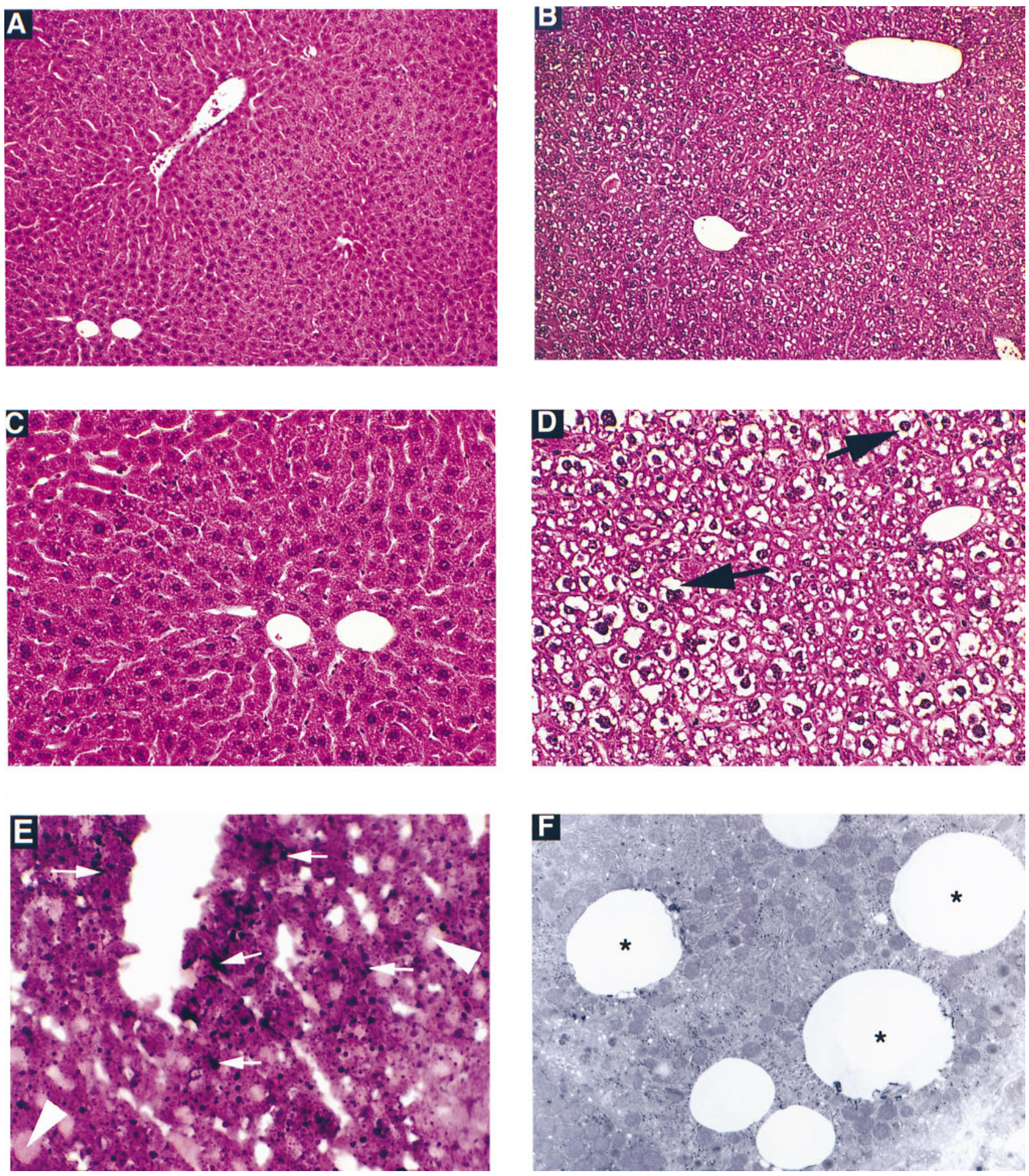

Figure 4. Hepatic changes in $H G F L^{-/-}$mice. H\&E staining of histological sections of livers from $H G F L^{+/+}(A$ and $C)$ and $H G F L^{-/-}(B$ and $D)$ mice showing cytoplasmic vacuolizations in hepatocytes of $H G F L^{-1-}$ mice (arrows point to vacuoles in hepatocytes). In $E$, arrows show lipid accumulation in cytoplasmic vacuoles of hepatocytes from HGFL-deficient mice, as demonstrated with Sudan black staining; arrowheads point to unstained nuclei. Transmission electron micrograph shows clear cytoplasmic droplets that are not membrane-bound in a background of normalappearing organelles (* in $F$ ). $A$ and $B, 100 \times ; C$ and $D, 200 \times ; E, 400 \times ; F, 3150 \times)$.

detectable anatomohistopathologic abnormality in $H G F L^{-1-}$ mice is lipid-filled cytoplasmic vacuolization of hepatocytes, which has no appreciable impact on unchallenged liver function.

Based on the ability of HGFL to stimulate differentiation of megakaryocytes and bone marrow mononuclear cells (20, 21 ), we also assessed the bone marrow precursors and the number and morphology of circulating blood cells in blood samples from groups of eight mice for each genotype. Myelocytic, erythrocytic, and megakaryocytic lineages were similar 


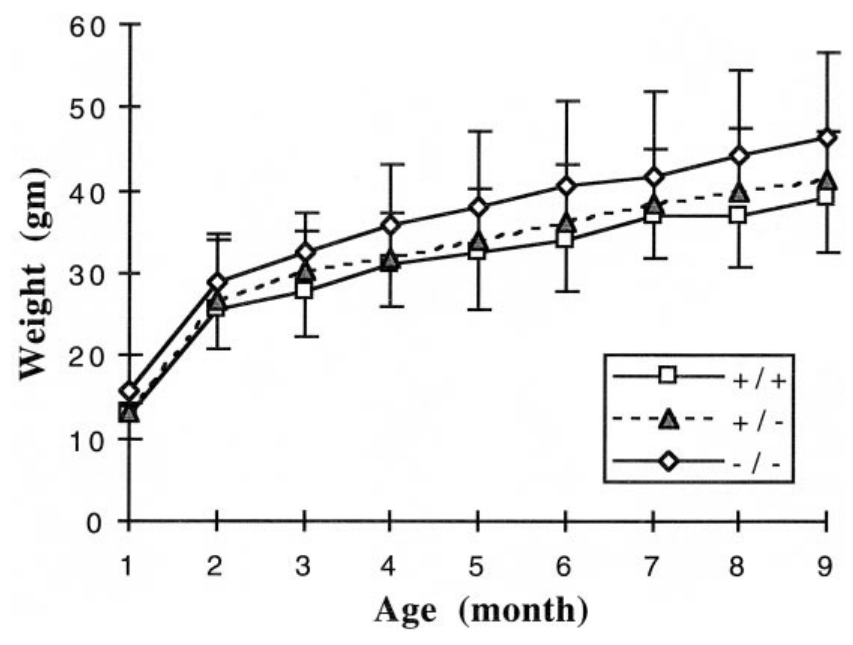

Figure 5. Growth of $\mathrm{HGFL}^{-/-}$mice. Longitudinal data showing similar weight gain in grams for $\mathrm{HGF}^{+/+},{ }^{+/}$, and ${ }^{-/-}$littermates followed up to 9 mo of age in the same room with the same caregivers. Each group represents the mean body wt of eight male and eight female mice for each one of the three genotypes; there is no statistical significance for body wt among groups.

in bone marrow aspirate from both $\mathrm{HGFL}^{+/+}$and ${ }^{-/-}$mice. Furthermore, there was no difference in the number or morphology of circulating erythrocytes, leukocytes (neutrophils, lymphocytes, monocytes, eosinophils, basophils), or platelets in $\mathrm{HGFL}^{-/-}$mice when compared with $H G F L^{+/-}$or ${ }^{+/+}$littermates (data not shown). Therefore, loss of HGFL was not associated with an appreciable monocytopenia or thrombocytopenia, although we cannot yet exclude the possibility that changes might become evident in older animals, or in the presence of biological challenges that stimulate the bone marrow of $H G F L^{-1-}$ mice.

Survival and fertility of $\mathrm{HGFL}^{-1-}$ mice. Cohorts of 16 mice for each HGFL genotype $(+/+,+/-,-/-)$ were followed by external examination and weekly weight measurements for 9 mo with no obvious phenotypic abnormalities. No differences have been observed with regard to either outward appearance, survival, or weight gain (Fig. 5). Productive crosses between $H G F L^{-/-}$male and $H G F L^{-/-}$female mice have shown that males are fertile, and females carry pregnancies to term with comparable litter size to wild-type controls. Generation of multiple $H G F L^{-/-}$offspring from $H G F L^{-/-}$male and female mice suggests that HGFL is not essential for spermatogenesis or fertilizing capacity in the epididymus (39).

Activation of peritoneal macrophages in $\mathrm{HGFL}^{-/-}$mice. To assess the role of HGFL in maturation of resident peritoneal macrophages, we performed morphometric analyses on macrophages obtained from peritoneal lavages of eight mice for each genotype. The number and morphology of resident peritoneal macrophages were similar in both $\mathrm{HGFL}^{+/+}$and $\mathrm{HGFL}^{-/-}$mice, demonstrating that HGFL is not essential for population of the peritoneal cavity by macrophages. We then assessed in vitro activation of macrophages in the absence of HGFL by incubating resident peritoneal macrophages from $\mathrm{HGFL}^{+/+}$mice with serum from $\mathrm{HGFL}^{+/+}$or $\mathrm{HGFL}^{-/-}$mice. Although activation of macrophages occurred normally in the presence of serum from $H G F L^{+/+}$mice, activation of mac- rophages was impaired in the first $13 \mathrm{~h}$ of incubation with serum from $\mathrm{HGFL}^{-/-}$mice, followed by a gradual increase in activation reaching comparable levels to those seen in $H G F L^{+/+}$ mice by $21-24 \mathrm{~h}$ (Fig. $6 \mathrm{~A}$ ). These data suggested that although HGFL potentiates early activation of peritoneal macrophages, other serum factors are ultimately sufficient to support full activation of macrophages in vitro. To test this hypothesis in vivo, we assessed migration of mononuclear phagocytes into the peritoneal cavity and activation of peritoneal macrophages $72 \mathrm{~h}$ after peritoneal injection of thioglycollate in $\mathrm{HGFL}^{+/+}$ and $\mathrm{HGFL}^{-/-}$mice. The total number $\left(4.9 \pm 1.8 \times 10^{6}\right.$ vs. $3.3 \pm 1 \times 10^{6}$ cells, $\mathrm{HGFL}^{+/+}$and ${ }^{-/-}$mice, respectively; $N=6$ for each genotype, $P=0.086$ ) and the percent of activation of peritoneal macrophages were similar in both $H G F L^{+/+}$and ${ }^{-/-}$

$\mathbf{A}$



B

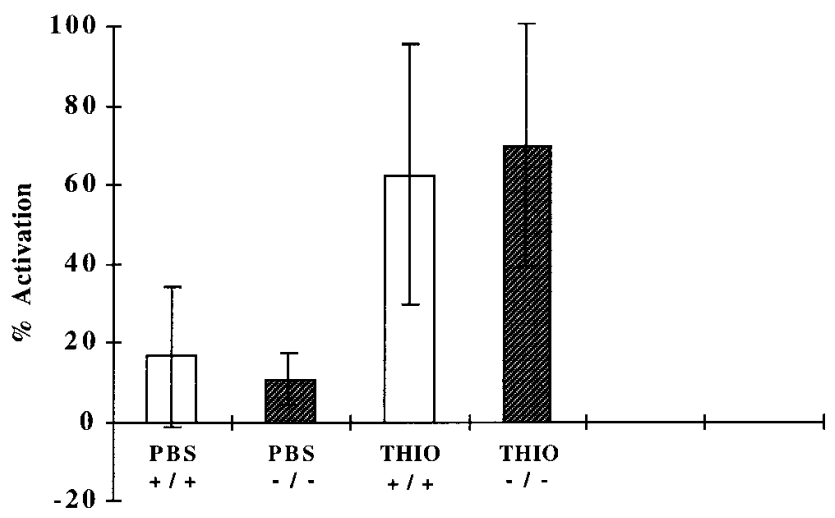

Figure 6. Activation of peritoneal macrophages in $\mathrm{HGFL}^{-/-}$mice. (A) Delayed in vitro activation of peritoneal macrophages in the absence of HGFL. Macrophages from wild-type mice were incubated with RPMI 1640 medium $(0 \%)$ or with medium containing $10 \%$ serum from $\mathrm{HGFL}^{+/+}$or $\mathrm{HGFL}^{-/-}$mice, and the percent of activation was determined at different time points using a reverse-phase microscope. Each time point represents the average percentage $( \pm \mathrm{SD})$ of activated macrophages of four different wells analyzed throughout the study. (B) In vivo activation of macrophages in $H G F L^{-/-}$mice. The average percentage $( \pm \mathrm{SD})$ of activated macrophages is plotted for each group $(N=8)$ of $H G F L^{+/+}$and $H G F L^{-/-}$mice after intraperitoneal injection of PBS or thioglycollate. Percent activation was determined after peritoneal macrophages were plated in wells of 24well culture dishes and incubated with serum-free RPMI for $30 \mathrm{~min}$. 




Figure 7. Healing of excisional skin wound in $H G F L^{-1-}$ mice. The percent of mice with open wounds plotted at different time points after a full-thickness incisional wound to the skin demonstrated similar wound healing in $\mathrm{HGFL}^{+/+}$and $H G F L^{-/-}$mice $(N=8$ for each group). Closure or healing of the wound was defined as closure of the incision and reappearance of epithelial surface.

mice (Fig. $6 \mathrm{~B}$ ), which suggests that activation of peritoneal macrophages can be achieved in vivo in the absence of HGFL.

Wound healing. To assess the impact of the in vivo loss of HGFL on wound healing, we examined the appearance of a linear incision to the skin of $\mathrm{HGFL}^{+/+}$and $\mathrm{HGFL}^{-/-}$mice daily until the epithelial surface was restored. All incisions had well-separated edges with exposure of the underlying muscle fascia at the time of surgery. There was minimal bleeding from the incision at the time of surgery, which resolved spontaneously within $5 \mathrm{~min}$ in all animals. Serial examination of the wound showed that the reparative pattern was characterized by formation of a dehydrated wound crust, gradual loss of the crust, appearance of a thin residual skin defect, closure of the incision surface, and reappearance of the epithelial surface in animals. These different phases of wound healing and the timing of repair was similar in $\mathrm{HGFL}^{+/+}$and $\mathrm{HGFL}^{-/-}$mice, with complete healing occurring between 11-15 d after surgery, suggesting that the outcome of wound repair after an incisional wound is not influenced by HGFL (Fig. 7).
Response to acute colitis. Although the sites of prenatal and postnatal mRNA expression for HGFL and ron/stk are predominantly in the gastrointestinal tract, there was no evidence of histopathologic abnormality along the digestive tract in $\mathrm{HGFL}^{-/-}$mice. These findings and the normal weight gain and stooling pattern of $\mathrm{HGFL}^{-1-}$ mice indicate that development of the intestinal tract occurred normally, and absorptive function remained unaltered in the absence of the protein. Based on expression of ron/stk in the epithelium of the stomach, small intestine, and colon, and on the cytokine properties of HGFL as a stimulator of macrophages and inhibitor of iNOS $(3,16-18,29)$, we assessed the potential role of HGFL in tissue repair after an inflammatory injury to the gastrointestinal tract. To accomplish this, we induced colitis in both $\mathrm{HGFL}^{+/+}$and $\mathrm{HGFL}^{-/-}$mice with administration of $5 \%$ dextran sulfate sodium in drinking water for $7 \mathrm{~d}$. Dextran sulfate sodium affects the intestinal epithelial cells and induces colonic inflammation with involvement of inflammatory mediators (40). As can be seen in Table I, although $\mathrm{HGFL}^{-1-}$ mice tended to be more affected by dextran sulfate sodium (diarrhea, visible blood in stools, weight loss), there was no appreciable difference in these parameters or in colonic histology (data not shown) at day 7 when compared with $H G F L^{+/+}$ mice.

\section{Discussion}

Our data demonstrate that the loss of HGFL in targeted mice allows for normal development to term, growth to adulthood, and reproduction. Furthermore, normal appearance, behavior, and long-term weight gain indicate adequate multiorgan function. This is in contrast to the embryonic lethal phenotype and the defects in the liver and placenta observed in mice lacking HGF $(5,6)$. Although HGF and HGFL belong to the same family of proteins and share similar in vitro biological functions such as growth and differentiation factors, our study clearly shows that they have different in vivo functions. It is possible that partial functional overlap of related proteins or binding of a homologous ligand to the receptor stk in $H G F L^{-/-}$ mice may compensate for the absence of HGFL (41). Since the receptors for HGFL and HGF share structural homology, HGF is a potential candidate to offset the lack of HGFL.

Table I. Induction of Acute Colitis by Oral Administration of 5\% Dextran Sulfate Sodium in $\mathrm{HGFL}^{+/+}$and $\mathrm{HGFL}^{-/-} \mathrm{Mice}^{-}$

\begin{tabular}{|c|c|c|c|c|c|c|c|}
\hline & Genotype & Day 1 & Day 2 & Day 3 & Day 4 & Day 5 & Day 6 \\
\hline \multirow[t]{2}{*}{ Hemoccult positive } & $+/+$ & $0 / 8$ & $0 / 8$ & $8 / 8$ & $8 / 8$ & $8 / 8$ & $8 / 8$ \\
\hline & $-1-$ & $0 / 8$ & $0 / 8$ & $8 / 8$ & $8 / 8$ & $8 / 8$ & $8 / 8$ \\
\hline \multirow[t]{2}{*}{ Visible blood } & $+/+$ & $0 / 8$ & $0 / 8$ & $0 / 8$ & $5 / 8$ & $8 / 8$ & $8 / 8$ \\
\hline & $-1-$ & $0 / 8$ & $0 / 8$ & $2 / 8$ & $7 / 8$ & $8 / 8$ & $8 / 8$ \\
\hline \multirow[t]{2}{*}{ Diarrhea } & $+1+$ & $0 / 8$ & $0 / 8$ & $0 / 8$ & $2 / 8$ & $2 / 8$ & $5 / 8$ \\
\hline & $-1-$ & $0 / 8$ & $0 / 8$ & $0 / 8$ & $3 / 8$ & $3 / 8$ & $7 / 8$ \\
\hline \multirow[t]{2}{*}{ Weight loss } & $+1+$ & $0.6 \pm 3$ & $+0.2 \pm 4$ & $1.8 \pm 4$ & $3.9 \pm 4$ & Not done & $9.5 \pm 9.6$ \\
\hline & $-1-$ & $5.5 \pm 11$ & $5.1 \pm 11$ & $7.5 \pm 11$ & $11 \pm 11$ & Not done & $16 \pm 15$ \\
\hline \multirow[t]{2}{*}{ Mortality } & $+1+$ & $0 / 8$ & $0 / 8$ & $0 / 8$ & $0 / 8$ & $0 / 8$ & $0 / 8$ \\
\hline & $-1-$ & $0 / 8$ & $0 / 8$ & $0 / 8$ & $0 / 8$ & $0 / 8$ & $1 / 8$ \\
\hline
\end{tabular}

Mice were examined daily, and stool samples were examined for the presence of gross (visible) or microscopic (Hemoccult ${ }^{\mathrm{TM}}$ ) blood and for diarrhea (defined as the presence of watery stools with or without blood). Data for each variable are expressed as the number of affected mice over the total number $(N=8)$ of mice in each group; weight loss is reported as the percent change of daily weight in comparison with the weight at the beginning of the study (day zero). 
HGF, however, is not capable of binding to or inducing phosphorylation of $s t k(18,24)$. Furthermore, targeted loss of $s t k$ in mice also allows for normal development and viability, which supports our findings that HGFL is not essential for embryogenesis, growth, or fertility (42).

Detailed gross and microscopic examination of tissues from $\mathrm{HGFL}^{-1-}$ mice does not reveal abnormalities in any organ, with the exception of the liver, where vacuolization of hepatocytes is found in the majority of $H G F L^{-/-}$mice. These hepatocytes contain intact cytoplasmic organelles, but the presence of small membrane debris and lipid-filled vacuoles suggest that they probably represent nonspecific signs of cellular injury. It is intriguing that a few $H G F L^{-/-}$mice have normal liver histology, and that similar histological findings were not apparent in stk-deficient mice (42). One variable that can account for such a discrepancy is the different genetic backgrounds used to generate $H G F L^{-/-}$and stk-deficient mice $(129 \mathrm{SvJ} / \mathrm{Swiss}$ Black vs. CD-1), a factor previously shown to influence the phenotype produced by targeted genes (43-45). This variable will be addressed by generation of $\mathrm{HGFL}^{-/-}$mice in different genetic backgrounds. An additional factor is an infectious process, but serological tests for common murine viruses have been repeatedly negative. The mechanistic linkage between these organelles and absence of HGFL remain to be established. Similar vacuolization, but of nuclear localization, was present in mice carrying targeted loss of the epidermal growth factor receptor (44). These mice also had thickened hepatocyte cords and distorted lobular architecture, and died before reaching adulthood. These findings were not seen in HGFL-deficient mice. Whatever the cause of vacuole formation in $\mathrm{HGFL}^{-/-}$ mice, these lesions do not significantly diminish unchallenged liver function based on serum biochemical markers and the overall good health of these mice. They do, however, provide a framework for future experiments that can directly assess the hepatic response to toxic, inflammatory, and infectious stimuli in the absence of HGFL.

Generation of viable $\mathrm{HGFL}^{-/-}$mice enabled us to define better the role of HGFL in hematopoiesis, differentiation, activation of macrophages, and tissue repair. Although a recent study has pointed to a key role of HGFL in differentiation of megakaryocytes (20), studies in $H G F L^{-/-}$mice showed no apparent defect in hematopoiesis, as evidenced by normal numbers and morphology of circulating blood cells (e.g. platelets). HGFL has been shown to potently activate resident peritoneal macrophages, inducing the formation of cytoplasmic projections and condensation of nuclear contents, chemotaxis, and phagocytosis of opsonized particles. However, it appears not to induce similar changes in circulating monocytes or inflammatory (exudate) macrophages, suggesting that HGFL may be important for tissue-specific maturation of resident peritoneal macrophages $(3,4,12,46)$. Two consequences of this activated state are release of proteases and induction of plasma membrane and cytoplasmic protein realignment, which are involved in effective migration of mononuclear cells toward inflammatory stimuli. Early steps of these processes appear to be impaired in the absence of HGFL, as demonstrated by delayed activation of resident peritoneal macrophages incubated with serum from $\mathrm{HGFL}^{-1-}$ mice. Absence of HGFL, however, did not compromise the final outcome since the same degree of activation of macrophages was achieved after longer incubation periods. In vivo, activation and recruitment of macrophages to sites of inflammation after injection of thioglycollate in
$\mathrm{HGFL}^{-/-}$mice proceeded unaltered by the absence of HGFL. These data suggest that one or more early steps in macrophage activation are dependent on HGFL, but, in the absence of the protein, other biochemical cues are sufficient ultimately to complete the activation process.

Based on the ability of HGFL to activate macrophages and trigger proliferation of keratinocytes, and based on its activation by proteases involved in tissue repair, HGFL has been suggested to play a role in wound healing and in the tissue response to inflammatory challenges (13-15). The reparative response to a skin wound is a complex tissue-remodeling process that involves formation of a matrix in the wound accompanied by infiltration of inflammatory cells and fibroblasts, proliferation and migration of keratinocytes through the provisional matrix, and contraction of the wound (47). The timely closure of an incisional skin wound in $\mathrm{HGFL}^{-/-}$mice, however, demonstrates that HGFL is not essential for wound healing, although our data do not preclude a potential role for the protein as an important mediator in the early steps after skin injury. The absence of HGFL also does not appear to influence the outcome of mice subjected to acute chemically induced colitis. Both $H G F L^{+/+}$and $H G F L^{-/-}$mice demonstrate similar clinical (e.g. diarrhea, weight loss) and histologic involvement of the gastrointestinal tract after a 7-d administration of dextran sulfate sodium, suggesting that although HGFL may aid in the local response to injury, HGFL is not essential for tissue repair and remodeling. This result is of particular interest based on mRNA expression of stk in the colon, and on the ability of HGFL to stimulate proliferation of colonic epithelial cells in culture $(3,16-18,25,29)$. Additional studies using $\mathrm{HGFL}^{-1-}$ mice will be important to assess the role of HGFL in the gastrointestinal mucosa response to infectious or other inflammatory processes (e.g. chronic toxic injury).

In conclusion, successful development of $\mathrm{HGFL}^{-1-}$ mice establishes fundamental biological differences from its homolog $H G F$. While the targeted loss of $H G F$ is embryonic lethal (with impaired liver development and placental defects), mice lacking HGFL demonstrate normal embryogenesis, growth, and fertility. Although HGFL-deficient mice have no obvious phenotypic abnormality, the presence of lipid-laden cytoplasmic vacuoles in hepatocytes points towards a potential role of HGFL in hepatic function. It will be of interest to assess whether these livers are able to regenerate after mechanical (partial hepatectomy) or toxic challenges. HGFL-deficient mice will also be valuable for studies concerning the cytokine properties of HGFL. Although our data suggest normal hematopoiesis and the presence of other in vivo compensatory mechanisms that are able to recruit and activate macrophages in the absence of the protein, it will be important to use HGFL-deficient mice to assess interaction of the protein with other cytokines and growth factors, and its role in the inflammatory response to infection, chronic tissue injury, and carcinogenesis.

\section{Acknowledgments}

The authors thank Dr. Kenn Holmbäk for assistance with the targeting experiments in ES cells, Dr. Thomas H. Bugge for help with the wound healing experiments, insightful discussions, and review of the manuscript, and Dr. William Balistreri for review of the manuscript.

This work was supported by the United States Public Health Ser- 
vice grants DK02341 (J.A. Bezerra) and DK47003 (S.J.F. Degen) from the National Institutes of Health.

\section{References}

1. Han, S., L.A. Stuart, and S.J.F. Degen. 1991. Characterization of the DNF15S2 locus on human chromosome 3: identification of a gene coding for four kringle domains with homology to hepatocyte growth factor. Biochemistry. 30:9768-9780.

2. Degen, S.J.F., L.A. Stuart, S. Han, and C.S. Jamison. 1991. Characterization of the mouse cDNA and gene coding for hepatocyte growth factor-like protein: expression during development. Biochemistry. 30:9781-9791.

3. Skeel, A.H., T. Yoshimura, D. Showelter, S. Tanaka, E. Appella, and E.J. Leonard. 1991. Macrophage stimulating protein: purification, partial amino acid sequence, and cellular activity. J. Exp. Med. 173:1227-1234.

4. Yoshimura, T., N. Yehki, M.-H. Wang, A. Skeel, and E.J. Leonard 1993. Cloning, sequencing and expression of human macrophage stimulating protein (MSP, MST1) confirms MSP as a member of the family of kringle proteins and locates the MSP gene on chromosome 3. J. Biol. Chem. 268:15461-15468.

5. Schmidt, C., F. Bladt, S. Goedecke, V. Brinkmann, W. Zschiesche, M. Sharpe, E. Gherardi, and C. Birchmeier. 1995. Scatter factor/hepatocyte growth factor is essential for liver development. Nature. 373:699-702.

6. Uehara, Y., O. Minowa, C. Mori, K. Shiota, J. Kuno, T. Noda, and N. Kitamura. 1995. Placental defect and embryonic lethality in mice lacking hepatocyte growth factor/scatter factor. Nature. 373:702-705.

7. Michalopoulos, G.K., and R. Zarnegar. 1992. Hepatocyte growth factor. Hepatology. 15:149-155.

8. Bardelli, A., C. Ponzetto, and P.M. Comoglio. 1994. Identification of functional domains in the hepatocyte growth factor and its receptor by molecular engineering. J. Biotechnol. 37:109-122.

9. Boros, P., and C.M. Miller. 1995. Hepatocyte growth factor: a multifunctional cytokine. Lancet. 345:293-295.

10. Bezerra, J.A., S. Han, M.J.S. Danton, and S.J.F. Degen. 1993. Are hepatocyte growth factor-like protein and macrophage stimulating protein the same protein? Protein Sci. 2:666-668.

11. Bezerra, J.A., D.P. Witte, B.J. Aronow, and S.J.F. Degen. 1993. Hepatocyte-specific expression of the mouse hepatocyte growth factor-like protein. Hepatology. 18:394-399.

12. Shimamoto, A., T. Kimura, K. Matsumoto, and T. Nakamura. 1993. Hepatocyte growth factor-like protein is identical to macrophage stimulating protein. FEBS Lett. 333:61-66.

13. Wang, M.-H., S.L. Gonias, A. Skeel, B.B. Wolf, T. Yoshimura, and E.J. Leonard. 1994. Proteolytic activation of single-chain precursor macrophagestimulating protein by nerve growth factor- $\gamma$ and epidermal growth factor-binding protein, members of the kallikrein family. J. Biol. Chem. 269:13806-13810.

14. Wang, M.-H., T. Yoshimura, A. Skeel, and E.J. Leonard. 1994. Proteolytic conversion of single chain precursor macrophage stimulating protein to a biologically active heterodimer by contact enzymes of the coagulation cascade. J. Biol. Chem. 269:3436-3440.

15. Wang, M.-H., A. Skeel, and E.J. Leonard. 1996. Proteolytic cleavage and activation of pro-macrophage-stimulating protein by resident peritoneal macrophage membrane proteases. J. Clin. Invest. 97:720-727.

16. Leonard, E.J., and A.H. Skeel. 1976. A serum protein that stimulates macrophage movement, chemotaxis and spreading. Exp. Cell Res. 102:434-438.

17. Wang, M.-H., G.W. Cox, T. Yoshimura, L.A. Sheffler, A. Skeel, and E.J. Leonard. 1994. Macrophage-stimulating protein inhibits induction of nitric oxide production by endotoxin- or cytokine-stimulated mouse macrophages. $J$. Biol. Chem. 269:14027-14031.

18. Gaudino, G., A. Follenzi, L. Naldini, C. Collesi, M. Santoro, K.A. Gallo, P.J. Godowski, and P.M. Comoglio. 1994. Ron is a heterodimeric tyrosine kinase receptor activated by the HGF homologue MSP. EMBO J. 13:3524-3532.

19. Wang, M.-H., A.A. Dlugosz, Y. Sun, T. Suda, A. Skeel, and E.J. Leonard. 1996. Macrophage-stimulating protein induces proliferation and migration of murine keratinocytes. Exp. Cell Res. 226:39-46.

20. Banu, N., D.J. Price, R. London, B. Deng, M. Mark, P.J. Godowski, and H. Avram. 1996. Modulation of megakaryocytopoiesis by human macrophagestimulating protein, the ligand for the RON receptor. J. Immunol. 156:2933: 2940 .

21. Kurihara, N., A. Iwama, J. Tatsumi, K. Ikeda, and T. Suda. 1996. Macrophage-stimulating protein activates stk receptor tyrosine kinase on osteoclasts and facilitates bone resorption by osteoclast-like cells. Blood. 87:37043710 .

22. Willett, C.G., D.I. Smith, V. Shridhar, M.-H. Wang, R.L. Emanuel, K. Patidar, S.A. Graham, F. Zhang, V. Hatch, D.J. Sugarbaker, and M.E. Sunday. 1977. Differential screening of a human chromosome 3 library identifies hepatocyte growth factor-like/macrophage-stimulating protein and its receptor in injured lung: possible implications for neuroendocrine cell survival. J. Clin. Invest. 99:2979-2991.
23. Wang, M.-H., C. Ronsin, M.-C. Gesnet, L. Coupey, A. Skeel, E.J. Leonard, and R. Breathnach. 1994. Identification of the ron gene product as the receptor for the human macrophage stimulating protein. Science. 266:117-119.

24. Wang, M.-H., A. Iwama, A. Skeel, T. Suda, and E.J. Leonard. 1995. The murine stk gene product, a transmembrane protein tyrosine kinase, is a receptor for macrophage-stimulating protein. Proc. Natl. Acad. Sci. USA. 92:39333937

25. Waltz, S.E., C.L.V. Toms, S.A. McDowell, L.A. Clay, R.S. Muraoka, E.L. Air, W.Y. Sun, M.B. Thomas, and S.J.F. Degen. 1998. Characterization of the mouse ron/stk receptor tyrosine kinase gene. Oncogene. 16:27-42.

26. Ponzetto, C., A. Bardelli, Z. Zhen, F. Maina, P.D. Zonca, S. Giordano, A. Graziani, G. Panayaotou, and P.M. Comoglio. 1994. A multifunctional docking site mediates signaling and transformation by the hepatocyte growth factor/ scatter factor receptor family. Cell. 77:261-271.

27. Medico, E., A.M. Mongiovi, J. Huff, M.-A. Jelinek, A. Follenzi, G. Gaudino, J.T. Parsons, and P.M. Comoglio. 1996. The tyrosine kinase receptors ron and sea control "scattering" and morphogenesis of liver progenitor cells in vitro. Mol. Biol. Cell. 7:495-504.

28. Collesi, C., M.M. Santoro, G. Gaudino, and P.M. Comoglio. 1996. A splicing variant of the ron transcript induces constitutive tyrosine kinase activity and an invasive phenotype. Mol. Cell Biol. 16:5518-5526.

29. Quantin, B., B. Schuhbaur, M.-C. Gesnel, P. Dolle, and R. Breathnach. 1995. Restricted expression of the ron gene encoding the macrophage stimulating protein receptor during mouse development. Dev. Dyn. 204:383-390.

30. van der Lugt, N., R.E. Maandag, H. te Riele, P.W. Laird, and A. Berns. 1991. A pgk:hprt fusion as a selectable marker for targeting of genes in mouse embryonic stem cells: disruption of the T-cell receptor $\delta$-chain-encoding gene. Gene. 105:263-267.

31. Mansour, S.L., K.R. Thomas, and M.R. Capecchi. 1988. Disruption of the proto-oncogene int- 2 in mouse embryo-derived stem cells: a general strategy for targeting mutations to non-selectable genes. Nature. 336:348-352.

32. Hooper, M.L., K. Hardy, A. Handyside, S. Hunter, and M. Monk. 1987. HPRT-deficient (Lesch-Nyhan) mouse embryos derived from germline colonization of cultured cells. Nature. 326:292-295.

33. Bugge, T.H., M.J. Flick, C.C. Daugherty, and J.L. Degen. 1995. Plasminogen deficiency causes severe thrombosis but is compatible with development and reproduction. Genes Dev. 9:794-807.

34. Reid, L.H., R.G. Gregg, O. Smithies, and B.H. Koller. 1990. Regulatory elements in the introns of the human HPRT gene are necessary for its expression in embryonic stem cells. Proc. Natl. Acad. Sci. USA. 87:4299-4303.

35. Li, H., D.P. Witte, W.W. Branford, B.J. Aronow, M. Weinstein, S. Kaur, S. Wert, G. Singh, C.M. Schreiner, J.A. Whitsett, et al. 1994. Gsh-4 encodes a LIM-type homeodomain, is in the developing nervous system and is required for early postnatal survival. EMBO J. 13:2876-2885.

36. Rømer, J., T.H. Bugge, C. Pyke, L.R. Lund, M.J. Flick, J.D. Degen, and K. Dano. Impaired wound healing in mice with a disrupted plasminogen gene. Nat. Med. 2:287-292.

37. Okayasu, I., S. Hatakeyama, M. Yamada, T. Ohkusa, Y. Inagaki, and R. Nakaya. 1990. A novel method in the induction of reliable experimental acute and chronic ulcerative colitis in mice. Gastroenterology. 98:694-702.

38. Cooper, H.S., S.N.S. Murthy, R.S. Shah, and D.J. Sedergran. Clinicopathologic study of dextran sulfate sodium experimental murine colitis. Lab. Invest. 69:238-249.

39. Ohshiro, K., A. Iwama, K. Matsuno, T. Ezaki, O. Sakamoto, I. Hamaguchi, N. Takasu, and T. Suda. 1996. Molecular cloning of rat macrophage-stimulating protein and its involvement in the male reproductive system. Biochem. Biophys. Res. Commun. 227:273-280.

40. Murthy, S.N., H.S. Cooper, H. Shim, R.S. Shah, S.A. Ibrahim, and D.J. Sedergran. 1993. Treatment of dextran sulfate sodium-induced murine colitis by intracolonic cyclosporin. Dig. Dis. Sci. 38:1722-1734.

41. Nowak, M.A., M.C. Boerlljst, J. Cooke, and J.M. Smith. 1997. Evolution of genetic redundance. Nature. 388:167-171.

42. Correll, P.H., A. Iwama, S. Tondat, G. Mayrhofer, T. Suda, and A. Bernstein. 1997. Increased interferon- $\gamma$-mediated nitric oxide production, in flammation and susceptibility to endotoxic shock in mice lacking the stk receptor tyrosine kinase. Genes \& Function. 1:1-15.

43. Miettinen, P.J., J.E. Berger, J. Meneses, Y. Phung, R.A. Pedersen, Z. Werb, and R. Derynck. 1995. Epithelial immaturity and multiorgan failure in mice lacking epidermal growth factor receptor. Nature. 376:337-341.

44. Threadgill, D.W., A.A. Dlugosz, L.A. Hansen, T. Tennenbaum, U. Lichti, D. Yee, C. LaMantia, T. Mourton, K. Herrup, R.C. Harris, et al. 1995. Targeted disruption of mouse EGF receptor: effect of genetic background on mutant phenotype. Science. 269:230-234.

45. Sibilia, M., and E.F. Wagner. 1995. Strain-dependent epithelial defects in mice lacking the EGF receptor. Science. 269:234-238.

46. Skeel, A., and E.J. Leonard. 1994. Action and target cell specificity of human macrophage-stimulating protein (MSP). J. Immunol. 152:4618-4623.

47. Martin, P. 1997. Wound healing: aiming for the perfect skin regeneration. Science. 276:75-81 